\title{
How implicitly activated and explicitly acquired knowledge contribute to the effectiveness of retrieval cues
}

\author{
Douglas L. Nelson, Serena L. Fisher, and Umit AKirmak \\ University of South Florida, Tampa, Florida
}

\begin{abstract}
The extralist cued recall task simulates everyday reminding because a memory is encoded on the fly and retrieved later by an unexpected cue. Target words are studied individually, and recall is cued by associatively related words having preexisting forward links to them. In Experiments 1 and 2, forward cue-to-target and backward target-to-cue strengths were varied over an extended range in order to determine how these two sources of strength are related and which source has a greater effect. Forward and backward strengths had additive effects on recall, with forward strength having a consistently larger effect. The PIER2 model accurately predicted these findings, but a plausible generation-recognition version of the model, called PIER.GR, could not. In Experiment 3, forward and backward strengths, level of processing, and study time were varied in order to determine how preexisting lexical knowledge is related to knowledge acquired during the study episode. The main finding indicates that preexisting knowledge and episodic knowledge have additive effects on extralist cued recall. PIER2 can explain these findings because it assumes that these sources of strength contribute independently to recall, whereas the eSAM model cannot explain the findings because it assumes that the sources of strength are multiplicatively related.
\end{abstract}

Seeing a word or phrase for the first time in a narrative can remind us of an earlier event. Similarly, grocery shopping can remind us of a dinner appointment made weeks earlier, or the sight of a neighbor's mailbox can remind us to return home to retrieve an overdue bill forgotten on the desk. In each example, a target event is encoded, and its recall is prompted by a retrieval cue that has been implicitly activated by the target event during its encoding. For example, writing the check that morning implicitly activates bill and associated concepts, such as envelope, stamp, post office, mail, and mailbox. Later, noticing a mailbox on the way to work effectively cues memory for the bill. The activation of such associates affects memory (e.g., Nelson, McKinney, Gee, \& Janczura, 1998), comprehension (e.g., Kintsch, 1988; Landauer \& Dumais, 1997), and behavior (Reich, Goldman, \& Noll, 2004; Stacy, 1997). Its effects are modeled in the laboratory by the extralist cuing task.

This article focuses on testing a model of how implicitly activated associates and explicit encoding operations (e.g., rehearsal) combine to constrain recall. In extralist cuing, familiar words or targets are presented one word at a time in the absence of specific semantic constraints. During testing, associated words are presented as retrieval cues for prompting target recall. The cuing is extralist because the cues are not presented with their targets during study. The task is ideal for investigating the effects of implicitly activated knowledge on recall because associated words are automatically recruited - for example, the target DINNER activates dinner and associated knowledge captured by such words as supper, lunch, and so on (e.g., Nelson et al., 1998). During testing, successful recall relies partly on associates activated by the target during study - for example, the cue LUNCH. Extralist cuing also works well for studying how the effects of implicitly activated associates and explicit encoding operations combine, because these two sources of information can be manipulated independently in this task.

Early work on extralist cuing indicated that the effectiveness of a test cue is a function of forward cue-to-target strength (e.g., Bahrick, 1969, 1970; Fox, Blick, \& Bilodeau, 1964) and backward target-to-cue strength (e.g., Humphreys \& Galbraith, 1975). Forward and backward strengths are measured by presenting individual words to large samples of participants asked to free associate the first word to come to mind, with strength determined by computing the probability of a given response to a particular cue (e.g., Bahrick, 1969; Cramer, 1968; Nelson, McEvoy, \& Schreiber, 2004). Free association probabilities provide a reliable index of mean relative strength of association in the absence of a controlled study trial (Nelson, Dyrdal, \& Goodmon, 2005; Nelson, McEvoy, \& Dennis, 2000). Hence, a cue with a strong forward link, a strong backward link, or both is likely to be more effective than one with weaker links (Nelson \& Goodmon, 2002). Forward links facilitate recall because they provide direct access to the target (Bahrick, 1969), and backward links

D. L.Nelson, dnelson@cas.usf.edu 
facilitate recall because they provide direct access from the target to the test cue (Thomson \& Tulving, 1970). The test cue, although physically absent during study, is likely to be implicitly present because of the preexisting backward link, and its activation constitutes a form of implicit encoding.

One specific aim of this article is to determine how the implicit activation of the test cue by the target contributes to the effectiveness of cues with forward links to their targets. Implicit processes mediate the effects of both forward and backward links. The issues are whether these links have additive or interactive effects on recall and whether one link is more important than the other. Experiments 1 and 2 crossed forward and backward strengths to determine how these links contribute their influence and which link is more important in producing correct recall. Prior research has indicated that backward strength adds its effects to forward strength (Nelson \& Goodmon, 2002), but in that study, strength was varied over a very narrow range ( $0-.13$ on a $0-1.0$ scale). Strong conclusions about the general relationship between forward and backward strength are unwarranted, because additive effects may not hold up at even moderate levels of strength. At higher levels, one source of strength may dominate the other. The relationship between forward and backward strength is theoretically important because classic extralist cuing explanations emphasize different links. For example, the two-phase model emphasizes the importance of the forward link in generating the target (Bahrick, 1970). In contrast, encoding specificity emphasizes the backward link by stressing the importance of encoding the test cue as a member of the target's set (Thomson \& Tulving, 1970; Tulving \& Thomson, 1973). Neither approach recognizes the potential importance of the link in the other direction.

In the PIER2 model ("processing implicit and explicit representations"; Nelson et al., 1998; Nelson, McEvoy, \& Pointer, 2003), both forward and backward links contribute to recall, but they make functionally separate contributions. In the model's computations, seeing a target word implicitly activates it and its associates in memory, and this activation differentially affects the relative influence of forward and backward strengths (see the Appendix for a numerical example). The effects of forward strength are weighted by the target's activation strength determined by its entire network of links. In contrast, the effects of backward strength are determined by the test cue's activation strength as an instance in the target's set. The effects of forward strength increase when the target's associative network is more richly interconnected, and the effects of backward strength increase when more of the target's associates are linked to the associate that becomes the test cue. The model computes the effects of forward and backward strengths indirectly by differentially weighting each source of information about the target.

These differences are illustrated numerically in Figure 1. Target activation strength is determined by adding its self-strength (1.0) to the sum of the strengths of the links making up its network. Regardless of origin or direction, target activation strength increases directly as a function

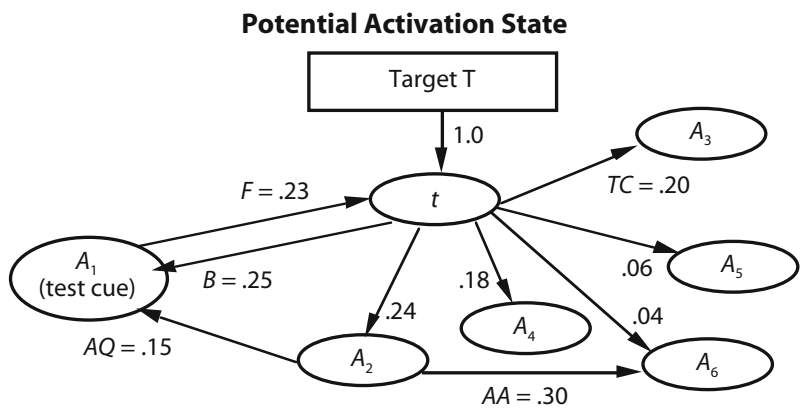

Activated State After Study

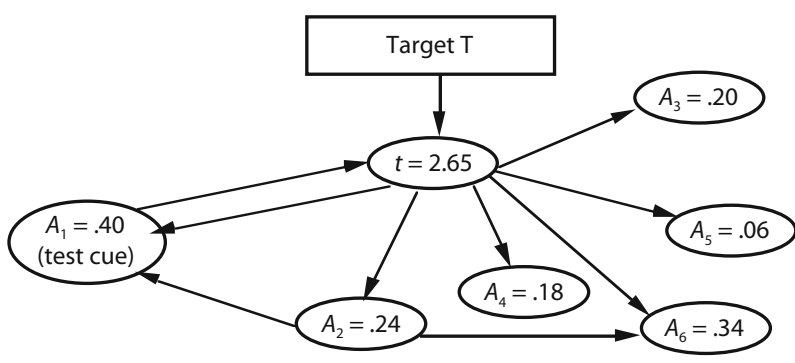

Predicted Recall

Assumes Cue Competitor Strength Is Zero and Target Competitor Strength Is $\left(A_{3}+A_{4}+A_{5}\right)=.44$

\begin{tabular}{|c|c|c|}
\hline $\begin{array}{c}\text { Cue-Target } \\
\text { Retrieval }\end{array}$ & $\begin{array}{c}\text { Activated } \\
\text { State of } t\end{array}$ & $\begin{array}{c}\text { Activated } \\
\text { State of } q\end{array}$ \\
\hline Target $(t)$ & 2.65 & .40 \\
\hline Test Cue $(q)$ & .23 & 1.00 \\
\hline
\end{tabular}

Implicit Target Strength Given $q=(.23 * 2.65)+(1.00 * .40)=1.01$

Predicted Recall of $t=1.01 \div(1.01+.44)=.70$

Figure 1. Activation state of the target before and after study processing, and a numerical example of how PIER2 computes probability of cued recall. Note $-t$, internal representation of the target; $A$, associate of the target; $F$, forward strength; $B$, backward strength; $T C$, target competitor; $A A$, associate-to-associate link; $A Q$, associate-to-cue link.

of the strength of its links. All links in the target's set are included, because prior research indicated that recall increases as associate-to-target and associate-to-associate strengths increase (e.g., Nelson et al., 2003). A summation rule is used because these two types of links have additive, rather than interactive, effects. Positive effects of associate-to-associate links do not depend on returning activation to the target on associate-to-target links. Target activation strength is important here because the model computes the effects of forward cue-to-target strength by multiplying it by target activation strength. In contrast to the target, test cue activation strength is increased by backward target-to-cue input and by input from the target's associates (see Associate $A_{1}$ in Figure 1). It is determined by adding backward strength to associate-to-cue strength, and in support of the model, research shows that recall increases as an additive function of these two sources (Nelson, Goodmon, \& Ceo, 2007). The model computes the effects of test cue activation by multiplying it by cue self-strength, set to 1.0. 
Given these rules for computing activation strength, PIER2 predicts that a given forward strength will have larger effects than will the same level of backward strength. Target activation is generally greater than test cue activation, averaging 4.35 (range, $1.45-11.43 ; n=$ 4,087 words) and 0.26 (range, $0-2.70$ ), respectively. Given equivalent forward and backward strengths, the multiplication of forward strength by target activation strength tends to produce a larger product than does cue activation strength multiplied by 1.0. Hence, the prediction that forward strength will generally have greater effects than will backward strength falls out of the model's computations.

The model also predicts when forward and backward strengths will have additive, as opposed to interactive, effects, because it makes a specific probability-of-recall prediction for each cue-target pairing. The model's predictions are pair specific. As is illustrated in Figure 1, the products of weighted forward and backward strengths are added together to determine the net implicit strength of a particular cue-target pair. Predicted probability of recall is then determined by dividing implicit strength by the sum of implicit strength plus competitor strength [PIER2 is a signal/(signal + noise) ratio model]. Competitors produce noise because they compete with the target and reduce its recall.

The second aim of this article is to determine how implicitly activated and explicitly encoded information combine to determine probability of recall. In PIER2, explicit encoding operations are independently applied to the target's internal representation in accordance with the study instructions and the experimental conditions. Operations such as those associated with intentional learning, rating concreteness, or naming vowels, to give some examples, produce an explicit or episodic representation of the target. Some operations are more effective than others, and the model assumes that the explicit representation varies in strength. What is more important here, the model assumes that the explicit representation independently adds encoding strength to the implicit representation of the target. PIER2 assumes that the target's net encoding strength is made up of the independent contributions of its implicit and explicit representations. Finally, the model assumes that these representations are encoded in a context and that context cues play a role in retrieving both encodings (Nelson et al., 2007).

In Experiment 3, forward and backward strengths, as well as explicit encoding strength, were varied by manipulating level of processing and study time. The key question concerns the relationship between implicit activation and explicit encoding strength: How does explicit strength contribute to the effectiveness of forward and backward links? The effects of explicit encoding strength may interact with the effects of forward and backward strength, or they may add their influence uniformly to the effects produced by such links. An interaction could show that cues with stronger preexisting links to their targets produce larger effects of level of processing and study time. If episodic strength has a larger effect when forward or backward strength is stronger, this finding could mean that recovering the explicit representation is contingent on recovering the implicit representation of the target. The extralist test cue recovers the target's implicit representation, which then recovers its explicit representation.

Alternatively, context cues may play a role in recovering both representations, as assumed (Humphreys, Tehan, O'Shea, \& Bolland, 2000; Mensink \& Raaijmakers, 1988; Nelson et al., 2007). Targets are encoded in the context of other list words in a specific place and time in the presence of an experimenter. External, social, and internal cues provide a context for the encoding experience, and such cues are known to affect free recall (see Smith \& Vela, 2001, for a review), as well as extralist cued recall (e.g., Nelson et al., 2007). If explicitly encoded information is recovered by context cues, rather than by the extralist cue, the effects of explicit encoding strength should add to the effects of forward and backward strength. Recall should be more likely when forward and backward strengths are greater, when processing is oriented toward meaning, and when more study time is available.

\section{EXPERIMENTS 1 AND 2}

The purpose of Experiments 1 and 2 was to cross forward and backward strengths at levels more extreme than those previously used in order to evaluate PIER2's predictions. The model predicts that recall will increase as forward and backward strengths increase; that forward strength will have larger effects than will backward strength; and that these strengths will have additive, rather than interactive, effects. Strengths in both experiments, regardless of direction, were in the ranges of .01-.10, .11-.20, and .21-.35 when strength was low, medium, and high, respectively. Link direction and strength were varied between subjects in Experiment 1, and in a mixed design, backward strength was varied within subjects in Experiment 2.

\section{Method}

Design and Participants. The design used for Experiment 1 formed a $3 \times 2$ between-subjects factorial involving forward strength (strong, medium, or weak) and backward strength (medium or weak). Strong backward strength could not be added to the design without confounding its manipulation with other variables being held constant. Cue-target pairs having strong forward and strong backward links are more rare than the other combinations. A withinlist manipulation of backward strength eliminated this problem, because fewer pairs are required. Hence, the design for Experiment 2 formed a $3 \times 3$ mixed-model factorial in which three levels of forward and of backward strength were varied, with backward strength manipulated within subjects. Both experiments were run to extend the generality of the findings, and two lists were created for each between-subjects condition. In Experiment 1, 26 participants were assigned to each of these conditions, with 12 assigned to each list. In Experiment 2, 24 participants were assigned in the same manner. All were assigned to conditions in randomized blocks and received points toward their grades in psychology courses for their service.

Materials for Experiment 1. Two 24-item lists were created for each of the six between-subjects conditions using free association norms (see Nelson et al., 2004). Regardless of direction, strength averaged $.27(S D=.05), .14(S D=.02)$, and $.04(S D=.03)$ for strong, medium, and weak levels, respectively. For example, forward and backward strengths in the strong-medium condition were $.26-.15$, whereas for the weak-weak condition, these strengths were .04-.05. Other variables known to affect extralist cued recall were held constant. Most important, target and cue activation strengths 
were equated within each strength condition. These controls are important because the effects of forward and backward links are manifested through the influence of different variables that must be controlled. The model multiplies forward strength by target activation strength, so target activation must be controlled in each forwardbackward strength condition. Similarly, the model adds backward strength to associate-to-cue strength and multiplies the sum by 1.0 , so associate-to-cue strength must be controlled in each forwardbackward strength condition. Target activation averaged $4.96(S D=$ $1.43)$, and associate-to-cue activation averaged $0.14(S D=0.20)$ across variations in forward and backward strength. Shared associate strength $(.03, S D=.05)$ and mediated strength $(.02, S D=.03)$ were controlled, as was target frequency (Kučera \& Francis, 1967), which averaged 95 words per million $(S D=140)$. Finally, cue and target competition effects were controlled for each strength manipulation. Given the cues that they were paired with, the targets generated $10.67(S D=4.07)$ competitors with average net target competitor strength of $.47(S D=.20)$. The test cues had $9.33(S D=4.32)$ competitors and an average net cue competitor strength of $.41(S D=$ .22 ). We used a multiple regression procedure to evaluate the success of controlling these features across both experiments, and this issue will be readdressed in the Results and Discussion section.

Materials for Experiment 2. Two 24-item lists were constructed for each level of forward strength, resulting in six different lists. Regardless of direction, mean strengths (and SDs) were $.27(.04)$, $.15(.03)$, and .04 (.03) for the strong, medium, and weak conditions, respectively. Target activation strength and associate-to-cue activation were controlled and averaged $4.64(S D=1.36)$ and 0.17 $(S D=0.26)$, respectively. Indirect shared associate and mediated strengths were $.04(S D=.06)$ and $.02(S D=.03)$. The targets were less frequent, as compared with those in Experiment 1, averaging 52 per million words $(S D=60)$, but they had similar levels of competitor strength. The targets produced $9.15(S D=3.71)$ competitors with an average target competitor strength of $.40(S D=.20)$, and the test cues had $8.60(S D=4.11)$ competitors and an average cue competitor strength of $.36(S D=.20)$.

Procedure. Data were collected in individual sessions. The participants were asked to read all the study words aloud and to remember as many words as possible without being told how they would be tested. Each word was presented for $3 \mathrm{sec}$ on a computer monitor, and prior to the study trial, a short list of names was shown to familiarize the participants with this rate. Immediately following the last study word, the participants were given the instructions for the test trial. The instructions indicated that cue words were going to be shown one at a time and that each cue would be meaningfully related to one of the studied words. The participants were asked to read each cue aloud and to recall its target orally, and they were told that they could guess when unsure. The test trial was self-paced, and the orders of appearance of both study and test words were unsystematically randomized for each participant.

\section{Results and Discussion}

ANOVA. Figure 2 shows the effects of forward and backward strength on probability of correct recall in Experiment 1 . Recall systematically increased as a monotonic function of forward strength and was affected by backward strength, with medium strength showing higher recall than did weak strength. Backward strength effects were nearly equivalent at each level of forward strength. This description is reinforced by the results of an ANOVA indicating that forward strength $\left[F(2,150)=24.75, M S_{\mathrm{e}}=\right.$ $.02]$ and backward strength $[F(1,150)=4.17]$ were significant sources of variance, whereas the interaction was not $(F<1)$. A planned least significant difference (LSD) of .06 indicated that recall significantly increased with each increment in forward strength. Similar results were

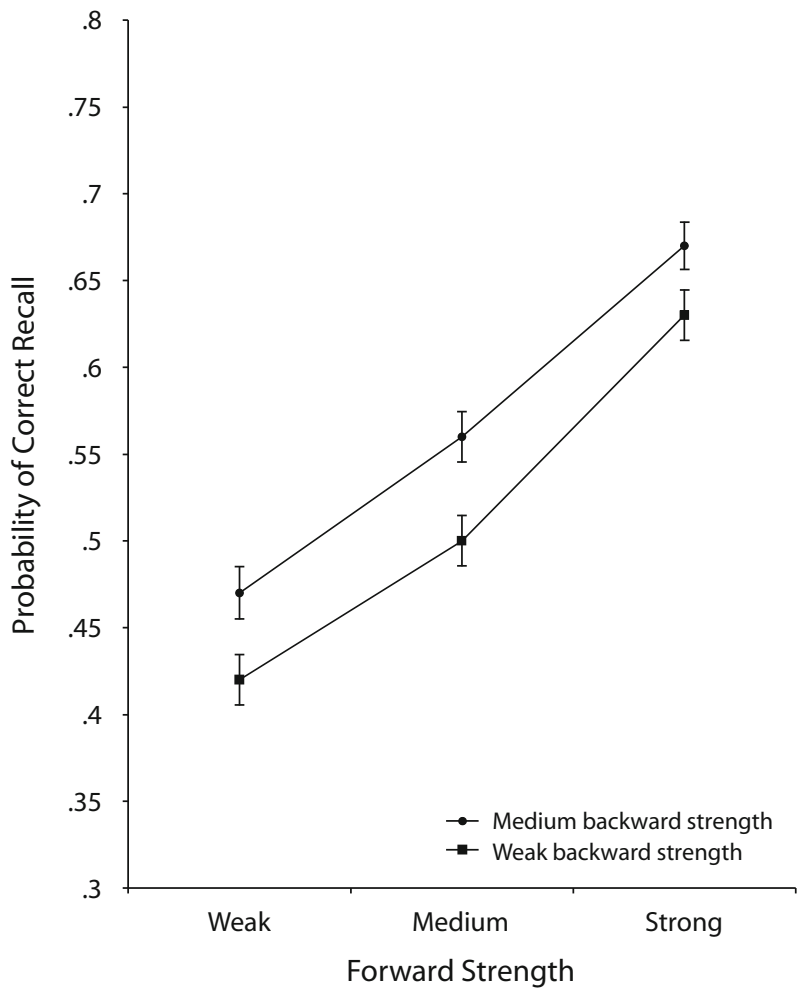

Figure 2. Probability of correct recall in Experiment 1 as a function of forward and backward strengths. Error bars are $S E$ s.

obtained when the strongest level of forward strength was omitted in the analysis. The effects of forward strength $\left[F(1,100)=8.96, M S_{\mathrm{e}}=.02\right]$ and backward strength $[F(1,100)=4.13]$ were significant, and the interaction was not.

The results of Experiment 2, shown in Figure 3, are similar. Probability of recall increases with forward and with backward strength, and although there is some instability in the medium backward strength condition, no interaction is apparent. The analysis indicated that the effects of forward strength $\left[F(2,69)=15.00, M S_{\mathrm{e}}=.06\right]$ and backward strength $\left[F(2,138)=4.23, M S_{\mathrm{e}}=.02\right]$ were significant, and the interaction was not $(F<1)$. Fisher's LSDs for forward and backward strengths are .08 and .05 , respectively. Regardless of whether backward strength is varied between or within subjects, it has additive, as opposed to interactive, effects on recall, and forward strength appears to have a greater effect on recall than does backward strength.

Regression analyses. Probability of correct recall for each cue-target pair was calculated in each experiment, and a database containing these probabilities and corresponding values for forward and backward strengths and other variables was constructed (see Nelson \& Zhang, 2000 , for details). These probabilities served as the dependent measure in a simultaneous multiple regression analysis, with forward and backward strengths, as well as the six variables held constant in these experiments, entered as predictors. The purpose of this analysis was to determine 


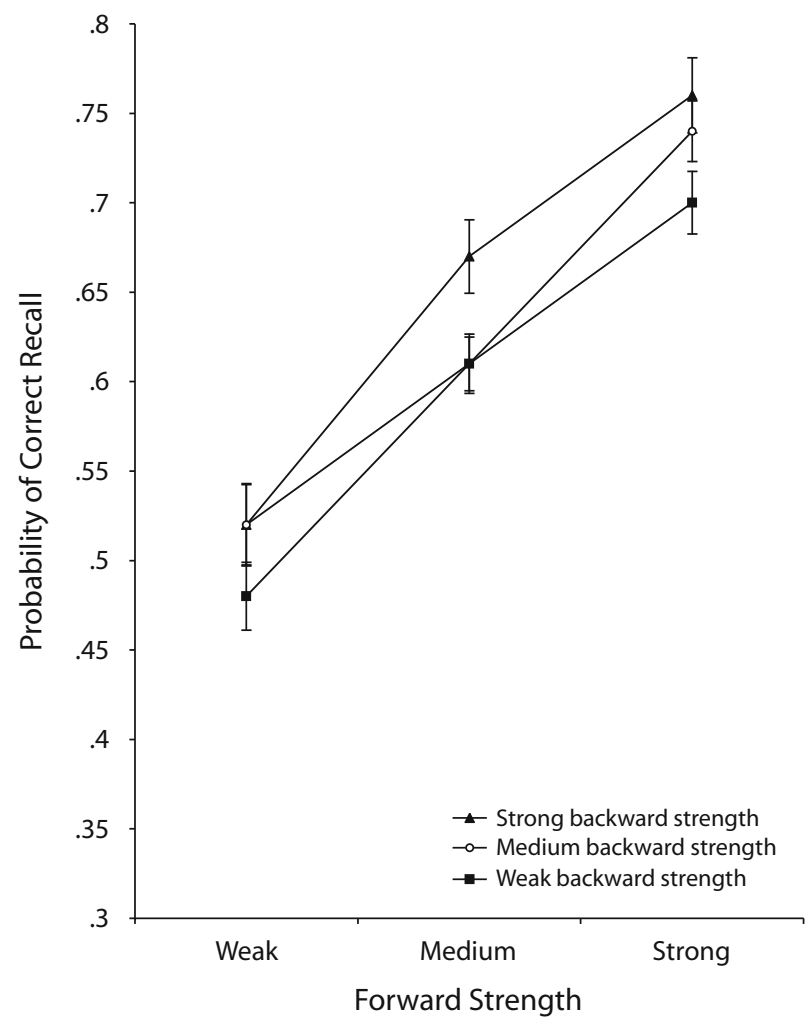

Figure 3. Probability of correct recall in Experiment 2 as a function of forward and backward strengths. Error bars are $S E s$.

whether forward and backward strength effects survive as predictors after statistically controlling for the other variables (see also Balota, Cortese, Sergent-Marshall, Spieler, \& Yap, 2004; Nelson \& Zhang, 2000). Complete data were available for 401 pairs, and the results of the regression were significant $\left[F(8,392)=20.65, M S_{\text {res }}=.04\right]$. Both forward strength and backward strength had significant beta coefficients: $.39(t=7.73, S E M=.11)$ and $.14(t=$ $2.95, S E M=.14)$, respectively. Effects of target frequency and target activation strength were also significant, but the effects of cue competitor, target competitor, shared associate, and mediator strengths were not. When the forward $x$ backward interaction was added as a predictor, it was not significant. A separate stepwise regression indicated that forward and backward strengths explained $14.8 \%$ and $3.3 \%$ of the variance, respectively. Hence, neither forward nor backward strength was dropped from the regression equation because of correlations with other variables. Forward strength had a larger effect than did backward strength, and these links had additive effects on recall.

Predicted recall probabilities. PIER2's predicted recall probabilities for each pair were computed for the two lists used in each strength condition, and they were subjected to the same types of ANOVAs as those used for the observed probabilities in each experiment. In calculating these predictions, only the implicit representation of the target was considered (Equation A5 in the Appendix).

The analysis of predicted probabilities indicated that the effects of forward strength were significant in each experiment $\left[F(2,252)=101.74, M S_{\mathrm{e}}=.028\right.$, in Experiment 1 , and $F(2,45)=13.17, M S_{\mathrm{e}}=.033$, in Experiment 2]. The effects of backward strength were also significant in each experiment $\left[F(1,252)=5.17, M S_{\mathrm{e}}=\right.$ .028 , in Experiment 1, and $F(2,90)=6.66, M S_{\mathrm{e}}=.029$, in Experiment 2]. The contributions of forward and backward strength were significant sources in each analysis, but the interactions were not $[F(2,252)=1.90$ in Experiment 1 , and $F(4,90)<1$ in Experiment 2]. The model predicts main effects of strength for each direction and that forward and backward strength will have additive effects on recall. Finally, the model predicts that the proportion of variance explained will be greater for forward strength ( $43 \%$ and $16.2 \%$ for Experiments 1 and 2, respectively) than for backward strength (1.4\% and $7.3 \%$, respectively). Hence, PIER2 predicted the qualitative pattern of recall found in each experiment.

To determine how well the model predicts the overall quantitative pattern, the data from the two experiments were pooled. Planned $t$ tests were used to compare predicted and obtained probabilities of recall as a function of forward and backward strengths. Observed (and predicted) probabilities of recall for forward strength are $.44(.40), .55(.55)$, and .67 (.70) for weak, medium, and strong cues, respectively. The model underestimates recall when forward strength is weak $[t(134)=2.02, p<$ $.04]$, but neither of the other two comparisons is significant. The observed (and predicted) probabilities for weak, medium, and strong backward strength are .48 (.46), .56 (.56), and .62 (.64), respectively. None of these differences are reliable.

Forward and backward strengths were varied over the widest feasible range given the need to control many variables, and the results indicate that they have additive effects on recall, as is predicted by PIER2 for the pairs used in these experiments. The model underestimates recall when forward strength is weak but otherwise predicts probability of correct recall accurately for each direction of strength. It also correctly predicts that forward strength will have a greater effect on recall than will backward strength when strength is varied at comparable levels on the same measurement scale. In the model, both types of links benefit from priming occurring during study, but the source of the priming differs, depending on whether it is based on the target or the cue as activated by the target. The effects of forward strength are determined by multiplying it by target activation level. In contrast, backward targetto-cue strength is added to associate-to-cue strength, and the results are multiplied by cue self-strength (1.0). The effects of backward strength are determined by test cue priming caused by the target and its associates, and this priming is recapitulated in full by the cue because it is presented during testing. In the model, target activation is greater than cue activation, so, for equivalent strengths, the forward cue-to-target link adds more to the predicted probability of cued recall than does the backward targetto-cue link. The prediction that forward strength will have a greater effect comes out of the math of the model's computations. However, note that the model also predicts exceptions to forward strength superiority. As manipulated 
forward strength approaches zero, the forward strength by target activation multiplication also approaches zero. Similarly, as target activation approaches cue activation, forward strength superiority is less likely to hold. The model predicts that backward strength superiority can emerge when either forward strength or target activation is exceptionally weak. The model can handle such exceptions to forward strength superiority because it makes pair-specific predictions on the basis of individualized computations of target and cue activation levels.

\section{EXPERIMENT 3}

Experiment 3 evaluated PIER2's assumption that both extralist and context cues drive the recovery of implicit and explicit representations (Nelson et al., 2007). Forward and backward strength were crossed in a factorial design with explicit encoding strength provided by level-of-processing and presentation rate manipulations. The key question was whether the effects of the explicit memory variables would interact with or add to the effects of the implicit memory variables. For example, if the effects of levels of processing and study time were more apparent when forward and backward strengths were stronger, this outcome would indicate that the effects of explicit encoding strength were contingent on the strength of the extralist cue. An interactive finding such as this one would imply that an extralist cue directly recovers the explicit representation, as well as the implicit representation. Alternatively, the effects of a stronger semantic encoding and more study time might be uninfluenced by the strength of the extralist cue. Additive effects imply that an extralist cue may recover the implicit, but not the explicit, representation. Context cues may facilitate the recovery of both representations, but they may serve as the primary and, perhaps, the only cues for recovering the explicit representation.

Because PIER2 assumes that implicit and explicit representations have independent effects in extralist cuing, it can explain both additive and interactive effects. The strength of the explicit encoding can add its effects to the strength of the implicit encoding, or these encodings can have interactive effects. The model can explain either outcome because recall can be based on the implicit representation, the explicit representation, or both representations. Findings showing additive effects would inform PIER2, but they cannot be regarded as an evaluation of the model, because the independence assumption allows for additive and multiplicative results. In contrast, the eSAM model uses a multiplicative sampling rule to combine the strengths of implicitly activated memories, explicit processing activities, and context (Sirotin, Kimball, \& Kahana, 2005). eSAM predicts that variations in the strengths of implicit and explicit memories will have interactive effects, so results showing additive effects would be inconsistent with this approach.

\footnotetext{
Method

Design and Participants. The design used for Experiment 3 formed a $2 \times 2 \times 2 \times 2$ between-subjects factorial involving forward strength (strong or weak), backward strength (medium or weak),
}

study orientation (rate concreteness or name vowels), and study time ( 2 or $6 \mathrm{sec}$ ). Eighteen participants served in each between-subjects condition, with 9 assigned to each list. All were assigned to conditions in randomized blocks, and they received points toward their grades for their service.

Materials and Procedure. The 24-pair lists used in Experiment 1 were also used here, but the medium forward strength condition was dropped in order to reduce the size of the design. Keeping the strong and weak forward strength conditions, rather than the medium and weak conditions, maximized the strength manipulation and increased the sensitivity of the design to potential interactive effects. The general procedures used in extralist cuing were also the same, with two exceptions. First, instead of presenting the targets for $3 \mathrm{sec}$ during study, each target was shown for either 2 or $6 \mathrm{sec}$. Second, instead of asking the participants to study and remember as many of the target words as possible, they were given incidental learning instructions. Half of the participants were asked to read the word aloud and then rate each word for concreteness on a 1-5 scale, with higher values representing higher concreteness. The remaining half were asked to read the word aloud and then name the vowels in the word as rapidly as possible. The participants were told that we were collecting norms for a long list of words, and memory testing was never mentioned. Immediately after the last study word, the extralist cuing instructions used for the prior experiments were provided.

\section{Results and Discussion}

ANOVA. Table 1 presents probability of correct extralist cued recall as a function of the four manipulated variables. As in Experiments 1 and 2, recall was higher when forward strength was higher (.63 vs. .41) and when backward strength was higher (.55 vs. .48). It was also higher after concreteness ratings than after vowel naming (.66 vs. .37) and when study was long as compared with short (.54 vs. .50). A four-factor ANOVA indicated that each of these variables had a significant effect on recall $\left[M S_{\mathrm{e}}=.017\right.$ for all sources, and, in the order reported, the $F$ values were $F(1,272)=210.03, F(1,272)=19.76$, $F(1,272)=341.85$, and $F(1,272)=7.23]$. This analysis also indicated that none of the interactions among these variables reached the .05 criterion for significance, with most $F \mathbf{s}<1$.

This experiment produced a very simple outcome, in that each variable significantly affected probability of extralist recall without interacting with any of the other manipulated variables. The critical interactions involving forward and backward strengths with level of processing and study time were not significant. Hence, the findings are consistent with the conclusion that the explicit represen-

Table 1

Probability of Correct Recall in Experiment 3 As a Function of Forward and Backward Strengths, Presentation Rate, and Levels of Processing

\begin{tabular}{|c|c|c|c|c|c|}
\hline \multirow[b]{3}{*}{$\begin{array}{l}\text { Forward } \\
\text { Strength }\end{array}$} & \multirow[b]{3}{*}{$\begin{array}{c}\text { Backward } \\
\text { Strength }\end{array}$} & \multicolumn{4}{|c|}{ Presentation Rate } \\
\hline & & \multicolumn{2}{|c|}{ Slow } & \multicolumn{2}{|l|}{ Fast } \\
\hline & & $\begin{array}{c}\text { Rate } \\
\text { Concreteness }\end{array}$ & $\begin{array}{l}\text { Name } \\
\text { Vowels }\end{array}$ & $\begin{array}{c}\text { Rate } \\
\text { Concreteness }\end{array}$ & $\begin{array}{c}\text { Name } \\
\text { Vowels }\end{array}$ \\
\hline Strong & $\begin{array}{l}\text { Medium } \\
\text { Weak }\end{array}$ & $\begin{array}{l}.80 \\
.74\end{array}$ & $\begin{array}{l}.55 \\
.50\end{array}$ & $\begin{array}{l}.81 \\
.72\end{array}$ & $\begin{array}{l}.46 \\
.46\end{array}$ \\
\hline Weak & $\begin{array}{l}\text { Medium } \\
\text { Weak }\end{array}$ & $\begin{array}{l}.64 \\
.54\end{array}$ & $\begin{array}{l}.31 \\
.22\end{array}$ & $\begin{array}{l}.58 \\
.45\end{array}$ & $\begin{array}{l}.26 \\
.23\end{array}$ \\
\hline
\end{tabular}


tation adds its effects to the effects of the implicit representation. Recovering the explicit representation appears to be independent of recovering the implicit representation, and its recovery seems likely to be driven by context cues (Humphreys et al., 2000; Nelson et al., 2007). This finding appears to be at odds with eSAM (Sirotin et al., 2005 ) and with the general assumption that the relationship between semantic and episodic memory is always multiplicative.

Regression analyses. Regression analyses were performed on the results of Experiment 3 and on an expanded version of the cued recall database reported in Nelson and Zhang (2000). These analyses explore the additive relationship between implicitly activated and explicitly elaborated memories at the level of specific cue-target pairs. Before turning to these analyses, a preliminary multiple regression analysis of the results of Experiment 3 indicated that the effects of the two implicit variables and the two explicit variables continued to affect recall significantly when the controlled variables were simultaneously entered in the regression as predictors. As in Experiments 1 and 2, effects of cue and target activation strength and other implicit variables were effectively controlled.

The additive relation issue was evaluated by entering PIER2 predictions (Equation A5 in the Appendix), level of processing, study time, and the interactions of these variables into a separate multiple regression equation. In PIER2, forward and backward strengths affect probability of recall indirectly after incorporating the influence of cue and target activation strength, competitor strength, and so on. The question is whether the effects produced by these implicit-processing variables at the level of the prediction equation interact with the effects of explicit-processing variables. The regression was significant $[F(5,682)=$ $104.94, M S_{\text {res }}=.048$, adj $\left.R^{2}=.43\right]$. PIER2's predictions, level of processing, and study time had significant effects on recall, with beta coefficients of $.44(t=15.17, S E M=$ $.03), .49(t=17.07, S E M=.02)$, and $.09(S E M=.02)$, respectively. When the interactions between PIER2's predictions with levels of processing and study time were entered into the regression equation, neither was significant ( $t=0.38$ for the levels interaction, and $t=0.62$ for the study time interaction). Hence, at the pair-specific level of analysis, the effects of implicit- and explicit-processing variables have additive effects on recall. Similar results were obtained when forward and backward strengths were entered as predictors in place of the PIER2 predictions.

Similar analyses were also run using a large cued recall database to determine whether the additive effects of the implicit- and explicit-processing variables would generalize over thousands of pairs taken from dozens of extralist cuing experiments. This database now contains probability of correct recall data for 4,067 cue-target pairs, along with predictors linked to implicit and explicit variables. The database is described in detail in Nelson and Zhang (2000), and both the earlier and the extended databases are available (luna.cas.usf.edu/ nelson/). Although 1,795 pairs taken from new cuing experiments have been added since the original database, the general characteristics of the data sets are similar. Testing was always self-paced, and most results were collected under intentional learning conditions with a 3 -sec target study time (2,802 pairs). Data were also collected under incidental learning conditions involving concreteness ratings (427 pairs), pleasantness ratings (95 pairs), vowel naming (139), and intentional conditions with different study times (604). The 10 variations in study conditions produced wide differences in recall; for example, for a 3-sec study time, recall after concreteness ratings, intentional learning, and vowel naming averaged $.68, .52$, and .39 , respectively.

To determine whether the two types of variables had additive or interactive effects in the larger database, a simultaneous multiple regression was run using PIER2 predictions and study condition as the only two predictors. The predictions varied widely as a function of differences in seven different implicit-processing variables. The first analysis was based on pairs studied for $3 \mathrm{sec}$ under one of three levels of explicit processing. Concreteness ratings, intentional learning, and vowel-naming conditions were assigned values of 3,2 , and 1 , respectively, reflecting their mean rank ordering on recall. The regression was significant $\left[F(2,3365)=772.94, M S_{\text {res }}=.048\right.$, adj $\left.R^{2}=.31\right]$. The implicit- and explicit-processing variables had significant beta coefficients: $.51(t=35.84$, SEM $=.02)$ and $.20(t=14.30, S E M=.01)$, respectively. More important, the two predictors were only weakly related (partial $r=$ -.06 ), and when the interaction between the implicit and the explicit predictors was added to the regression, it was not significant. The results were unaffected when all 10 learning conditions were ranked from highest to lowest mean recall and included in the data analysis in place of the three levels of processing. Hence, the relative contributions of implicit and explicit variables to recall in the extralist cuing task appear to be additive, not interactive. Additivity holds in Experiment 3 and in thousands of pairs taken from many individual experiments involving a wide range of encoding conditions.

\section{GENERAL DISCUSSION}

Experiments 1 and 2 go beyond demonstrating that extralist cued recall increases as forward cue-to-target strength and backward target-to-cue strength increase. Forward and backward strengths were varied over an extensive range, and within this range, the results show that backward strength adds its influence to the effects of forward strength. They also show that, at comparable levels of manipulated strength, forward strength contributes more to successful recall than does backward strength. A forward strength of .25 has a greater effect on recall than does a backward strength of .25. Experiment 3 shows that explicit-encoding operations applied to the target during study add their influence to the effects of both forward and backward strength. Recall in extralist cuing is higher when the preexisting links between the cue and the target are stronger, and recall is higher when encoding operations are directed toward target meaning and when study time is longer. For both forward and backward links, the effects of meaningful processing and more study time were just as apparent for stronger as they were for weaker cues. 
These findings place important constraints on any model of extralist cuing, and they add to our understanding of how retrieval cues in the environment serve as reminders for prompting recall. Such cues tend to be more effective when the information emanates from the test cue during retrieval than when it emanates from the target during encoding, but regardless of source, their influence on recall is additive. In addition, cues tend to be more effective when the target has been more strongly encoded because of explicit-processing operations, no matter how strong the cue is. Implicit and explicit sources of information have additive effects as well.

\section{Theoretical Implications of Forward and Backward Strength Effects}

The additive relation between forward and backward strengths is consistent with PIER2's predictions for the lists used in the present experiments. The model makes pair-specific predictions, and different lists may have predicted an interaction showing weaker effects of backward strength with increasing forward strength. In evaluating the model, the crucial result is not whether forward and backward strength have additive or interactive effects in the data analysis, but what the model predicts in relation to what the data show. The results of Experiments 1 and 2 confirm the model's predictions both qualitatively and quantitatively. With one exception, the quantitative predictions came very close to the expected values, even though the model estimated no parameters from the recall data. The model's success is derived solely from how it concatenates measured connection strengths between word pairs making up the networks of the studied target and its test cue.

In this context, it is important to understand that the model computes the relative influences of forward and backward strength indirectly. The effects of forward cueto-target strength are determined by multiplying forward strength by target activation strength. In contrast, the effects of backward target-to-cue strength are computed by adding backward strength to associate-to-cue strength and multiplying the sum by cue self-strength. The results of computing the effects of forward and backward strengths in this manner are then summed to determine net cuetarget strength. This computation seems complex, but it amounts to differentially weighting the effects of forward and backward strengths by what is implicitly activated during study. The effects of forward strength to a particular target increase when that target has a more richly connected associative network, and the effects of backward strength increase when more of the target's associates have preexisting links to the associate that becomes the cue. Because these two sources of strength are differentially weighted, the model predicts that forward strength effects will generally be greater than backward strength effects when the two sources have the same levels of nominal strength. Forward strength is predicted to have a greater effect because target activation strength is generally greater than associate-to-cue strength.

Earlier explanations of extralist cuing correctly identified the importance of forward (Bahrick, 1970) and back- ward (Thomson \& Tulving, 1973) links, but they could not explain the differential effects of forward strength on recall. Similarly, models that are more recent fail for the same reason. For example, WAS-REM computes cue-target strength by summing forward, backward, and mediated free association strengths (Steyvers, Shiffrin, \& Nelson, 2005). Although this computation is more successful in predicting recall than are measures based on co-occurrence norms, it cannot explain why the same experimental value of forward strength has a greater effect on recall than does the same level of backward strength. Differential effects of forward and backward strength cannot be explained by any model that weights each source of strength equally, and hence this result is theoretically important for discriminating between models of cued recall. As a case in point, this result eliminates a generationrecognition version of PIER2, called PIER.GR.

PIER.GR offers a plausible explanation for many findings and has an optional recognition process for checking list membership. PIER2 is a generation model that has no computationally defined recognition check for deciding whether a recovered item is a member of the study list. PIER.GR incorporates such a check, and the model is simpler than PIER2 because it computes generation and recognition processes in separate steps and then sums the results, as in SAM (search of associative memory; Gillund \& Shiffrin, 1984). In brief, generation strength is determined by summing forward, backward, and two-step strengths. Generation probability is then determined by dividing generation strength by the sum of generation, cue competitor, and target competitor strengths. As in PIER2, the probability of sampling the target in the presence of the test cue is determined by a ratio rule, but, in contrast to PIER2, PIER.GR computes generation likelihood independently of target activation. Forward and backward strengths enter the model directly, so forward strength is no longer weighted by target activation. Instead, target activation is assigned an independent role in recognition (Nelson, Zhang, \& McKinney, 2001). As in PIER2, target activation is computed by summing the link strengths for each pairwise connection in the target's set, and recognition probability is determined by dividing this sum by the number of links in the target's associative matrix. Probability of recall in extralist cuing is then determined by adding the probability of generation to the probability of recognition. Like PIER2, this model makes pair-specific predictions as a function of variations in target activation level, competitor effects, and forward and backward strengths. However, unlike PIER2, it treats forward strength and backward strength as equivalent sources of information. Equivalent values of forward and backward strengths are predicted to have equivalent effects on generation probability and, therefore, on recall. PIER.GR explains as well as PIER2 why different types of preexisting connections affect extralist cuing, but, among other things, it cannot explain why forward strength has a greater effect on recall.

Partly because of this shortcoming, PIER.GR underestimates probability of recall, as compared with PIER2. Figure 4 compares the predictions of the two models as a 
function of five categories of forward strength, using the extended cued recall database described earlier. Mean recall probabilities for forward strengths of $0-.10, .10-.20$, $.20-.30, .30-.40$, and .40 or above were based on 1,717 , $1,147,676,342$, and 185 cue-target pairs. As is shown in the figure, backward strength was not confounded with forward strength in these comparisons, because it remained constant. To simplify computations, the strength of the explicit representation was set to zero, and the retention interval was set to $1 \mathrm{~min}$. The predictions reflect the influence of only the implicit representation computed according to each model. Both models predict linear increases in recall with increasing levels of forward strength, so both models accurately shadow observed probability of correct recall. PIER.GR, however, underestimates probability of correct recall at each level of forward strength, whereas PIER2 fits reasonably well. ${ }^{1}$ Despite its plausibility, PIER.GR fails as a model of extralist cuing.

\section{Theoretical Implications of Implicit and Explicit Strength Effects}

PIER2 was designed to explain the effects that implicitly activated associates have on memory for recent experience, but it incorporates roles for explicit-processing activities and for the importance of context cues (Nelson et al., 2007). Although the explicit-processing aspects of the model remain undeveloped, it makes assumptions about how these three sources of information are related and speaks to the extremely important issue of how implicit-semantic and explicit-episodic memory are related. PIER2 assumes that implicit- and explicit-processing activities provide independent sources of knowledge about target encoding.

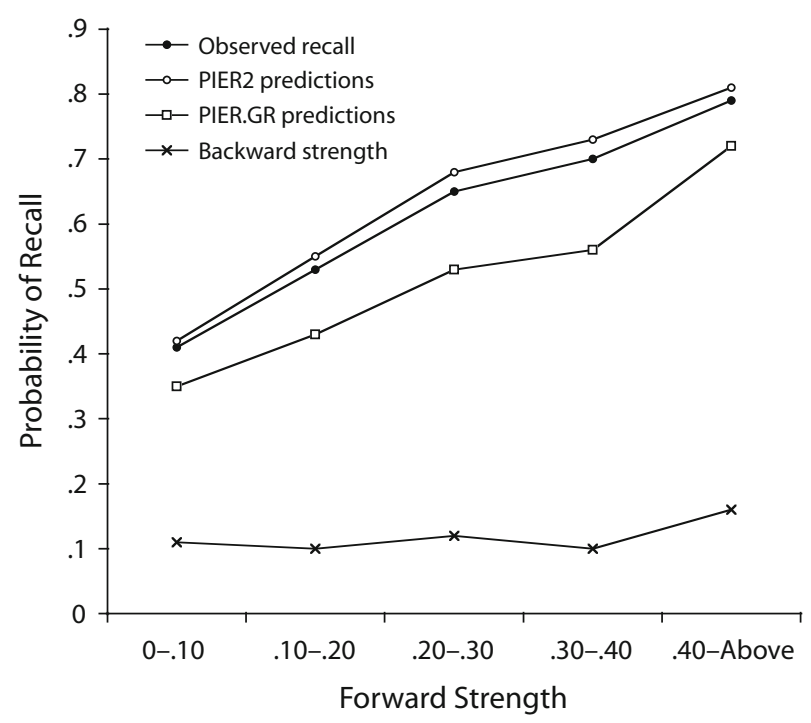

Figure 4. Observed and predicted probability of correct recall for PIER2 and PIER.GR as a function of forward strength. The plot of backward target-to-cue strength shows that this variable did not vary systematically with forward strength in the database. The $S E$ s are not shown in the figure; they were too small to appear because of the large sample sizes involved. For observed recall, PIER2 predictions, and PIER.GR predictions, they were $.010, .006$, and .009 , respectively.
Furthermore, the effects of both sources are modulated by delays and context disruptions occurring after study that reduce the accessibility of context information encoded during study (see the Appendix). Context is associated to both implicit- and explicit-processing activities, but the results of implicit and explicit processing have additive or interactive effects.

The findings of Experiment 3 and the regression findings based on thousands of cue-target pairs suggest that the results of implicit and explicit processing are more likely to be additive than interactive. These results carry interesting implications for PIER2 and eSAM. One is that recovering the explicit encoding of the target is not contingent on recovering the implicit representation. The effects of encoding strength produced because of elaborative encoding are not more apparent when the extralist cue is stronger by virtue of preexisting forward or backward links. Instead, the effects of meaningful encoding and more study time are uninfluenced by the strength of the extralist cue. The extralist cue appears to recover the implicit, but not the explicit, encoding of the target. Context cues facilitate the recovery of each encoding and may serve as the only cues for recovering the explicit representation.

If the additive influences of implicit and explicit encoding operations stand up under further evaluation, PIER2 can be expressed more simply by summing the effects of explicit (E) and implicit (I) encoding and weighting the sum by the effects of context accessibility (c) during testing. The general relationship between the probability of recalling target (T) given extralist (Q) and context (C) cues may be described as

$$
\operatorname{Pr}(\mathrm{T} \mid \mathrm{Q}, \mathrm{C})=\mathrm{E}+\mathrm{I}\left(t^{-c}\right) \text {. }
$$

In this equation, recall is a function of the sum of implicitand explicit-processing operations multiplied by time $t$ in minutes raised to a negative power of context accessibility $(c) . c$ is a parameter that varies with the nature of the disruption task that fills the retention interval, and it must be estimated from the data (Nelson et al., 2007). The equation causes the sum of the implicit and explicit encoding strengths to weaken as a power function of the length of the retention interval, with the rate of forgetting determined by the loss of access to information encoded about context.

The additive effects produced by implicit- and explicitprocessing operations also carry implications for eSAM, which was designed for predicting free recall findings in a way that incorporated the influence of preexisting semantic memories (Sirotin et al., 2005). The probability of sampling the target, given item and context cues, is computed as a multiplicative function of semantic, episodic, and context strengths. This multiplicative rule means that each type of association constrains the influence of the other associations on sampling probabilities. Each type of association is weighted so that the amount of constraint can be varied according to task and conditions, but each type of association always modulates the influence of the other types. The effects of episodic encoding strength always depend on semantic strength. 
Both PIER2 and eSAM represent extensions of the general retrieval rules developed by Raaijmakers and Shiffrin (1981). Both models address the relative influences of semantic, episodic, and contextual associations, both rely on using words and context as cues; and both weight semantic and episodic strength by context. However, because of the multiplicative rule, eSAM cannot easily explain how implicit-semantic and explicit-episodic strengths can have additive effects. Reducing the weight on episodic strength for the extralist cuing task does not resolve the problem. Reducing the influence of episodic strength also reduces the influence of semantic strength in a multiplicative model. Consequently, manipulations of implicit variables such as forward strength would be predicted to have reduced effects that would be more apparent when episodic strength is weak (e.g., after naming vowels) than when it is strong (e.g., after rating concreteness). Reducing the importance of the episodic component still leaves eSAM predicting an interaction between semantic and episodic strengths. Hence, eSAM cannot easily explain findings showing that levels of processing and study time add their effects to forward and backward strengths.

eSAM's hypothesized multiplicative relationship between semantic, episodic, and context associations may apply well to free recall, but not to extralist cued recall. The present findings limit the generality of eSAM, but in fairness, it is important to note that free and cued recall are very different memory tasks that involve different processes and that respond to the same variables in different ways. For example, for lists of unrelated words, printed frequency has different effects in free recall than in extralist cuing. High target frequency either benefits free recall or has no effect, but in extralist cuing, low-frequency words are uniformly more likely to be recalled than highfrequency words (e.g., Nelson \& Xu, 1995; Nelson \& Zhang, 2000). Similarly, target set size effects have opposite effects in free and cued recall. Target set size refers to how many relatively strong associates a word has, as indexed via free association norms. In the free recall task, words with larger sets of associates are more likely to be recalled than are words with smaller sets, whereas in the extralist cuing task, the opposite is true (Nelson, McEvoy, \& Schreiber, 1990). The free recall task relies heavily on making episodic associations between the words. Words with more associates are likely to have more mediating links that facilitate the creation and recall of episodic associations. In contrast, when cued with an extralist cue, targets with more associates generate more competitors, and they reduce the probability of recall.

In fairness to eSAM, PIER2 was explicitly designed for extralist cuing and cannot predict the set size effects found in free recall. Models of free recall and extralist cuing need to be different because these two tasks require very different types of processing during retrieval. An interactive sampling rule may work best in predicting free recall, whereas an additive rule for explicit and implicit information seems to work best for extralist cuing. The more important point here is that no single relationship between implicit-semantic knowledge and explicit- episodic knowledge is likely to hold across all tasks, especially when they are as different as free recall and extralist cued recall.

\section{AUTHOR NOTE}

This research was supported by Grant MH16360 from the National Institute of Mental Health to D.L.N. We especially thank Cathy McEvoy for comments on an earlier draft. Correspondence concerning this article should be addressed to D. L. Nelson, Department of Psychology, University of South Florida, PCD 4118G, Tampa, FL 33620-8200 (e-mail: dnelson@cas.usf.edu).

\section{REFERENCES}

BAHRICK, H. P. (1969). Measurement of memory by prompted recall. Journal of Experimental Psychology, 79, 213-219.

BAHRICK, H. P. (1970). Two-phase model for prompted recall. Psychological Review, 77, 215-222.

Balota, D. A., Cortese, M. J., Sergent-Marshall, S. D., Spieler, D. H., \& YAP, M. J. (2004). Visual word recognition of single-syllable words. Journal of Experimental Psychology: General, 133, 283-316.

Cramer, P. (1968). Word association. New York: Academic Press.

Fox, P. W., Blick, K. A., \& Bilodeau, E. A. (1964). Stimulation and prediction of verbal recall and misrecall. Journal of Experimental Psychology, 68, 321-322.

Gillund, G., \& Shiffrin, R. M. (1984). A retrieval model for both recognition and recall. Psychological Review, 91, 1-67.

Humphreys, M. S., \& Galbraith, R. C. (1975). Forward and backward associations in cued recall: Predictions from the encoding specificity principle. Journal of Experimental Psychology: Human Learning \& Memory, 1, 702-710

Humphreys, M. S., Tehan, G., O'Shea, A., \& Bolland, S. W. (2000). Target similarity effects: Support for the parallel distributed processing assumptions. Memory \& Cognition, 28, 798-811.

KINTSCH, W. (1988). The role of knowledge in discourse comprehension: A construction-integration model. Psychological Review, 95, 163-182.

KuČera, H., \& Francis, W. N. (1967). Computational analysis of present-day American English. Providence, RI: Brown University Press.

LANDAUER, T. K., \& Dumais, S. T. (1997). A solution to Plato's problem: The latent semantic analysis theory of acquisition, induction, and the representation of knowledge. Psychological Review, 104, 211-240.

MensinK, G.-J., \& RaAiJmaKers, J. G. W. (1988). A model for interference and forgetting. Psychological Review, 95, 434-455.

Nelson, D. L., Dyrdal, G. M., \& Goodmon, L. B. (2005). What is preexisting strength? Predicting free association probabilities, similarity ratings, and cued recall probabilities. Psychonomic Bulletin \& Review, 12, 711-719.

Nelson, D. L., \& Goodmon, L. B. (2002). Experiencing a word can prime its accessibility and its associative connections to related words. Memory \& Cognition, 30, 380-398.

Nelson, D. L., Goodmon, L. B., \& Ceo, D. (2007). How does delayed testing reduce effects of implicit memory: Context infusion or cuing with context? Memory \& Cognition, 35, 1014-1023.

Nelson, D. L., McEvoy, C. L., \& Dennis, S. (2000). What is free association and what does it measure? Memory \& Cognition, 28, 887-899.

Nelson, D. L., McEvoy, C. L., \& Pointer, L. (2003). Spreading activation or spooky action at a distance? Journal of Experimental Psychology: Learning, Memory, \& Cognition, 29, 42-52.

Nelson, D. L., McEvoy, C. L., \& Schreiber, T. (1990). Encoding context and retrieval conditions as determinants of the effects of natural category size. Journal of Experimental Psychology: Learning, Memory, \& Cognition, 16, 31-41.

Nelson, D. L., McEvoy, C. L., \& Schreiber, T. A. (2004). The University of South Florida free association, rhyme, and word fragment norms. Behavior Research Methods, Instruments, \& Computers, 36, 402-407.

Nelson, D. L., McKinney, V. M., Gee, N. R., \& Janczura, G. A. 
(1998). Interpreting the influence of implicitly activated memories on recall and recognition. Psychological Review, 105, 299-324.

Nelson, D. L., \& XU, J. (1995). Effects of implicit memory on explicit recall: Set size and word frequency effects. Psychological Research, 57, 203-214

Nelson, D. L., \& Zhang, N. (2000). The ties that bind what is known to the recall of what is new. Psychonomic Bulletin \& Review, 7, 604-617.

Nelson, D. L., Zhang, N., \& McKinney, V. M. (2001). The ties that bind what is known to the recognition of what is new. Journal of Experimental Psychology: Learning, Memory, \& Cognition, 27, 1147-1159.

RaAiJmakers, J. G. W., \& Shiffrin, R. M. (1981). Search of associative memory. Psychological Review, 88, 93-134.

Reich, R. R., Goldman, M. S., \& Noll, J. A. (2004). Using the false memory paradigm to test two key elements of alcohol expectancy theory. Experimental \& Clinical Psychopharmacology, 12, 102110.

Sirotin, Y. B., Kimball, D. R., \& Kahana, M. J. (2005). Going beyond a single list: Modeling the effects of prior experience on episodic free recall. Psychonomic Bulletin \& Review, 12, 787-805.

Smith, S. S., \& Vela, E. (2001). Environmental context-dependent memory: A review and meta-analysis. Psychonomic Bulletin \& Review, 8, 203-220.

StACY, A. W. (1997). Memory activation and expectancy as prospec- tive predictors of alcohol and marijuana use. Journal of Abnormal Psychology, 106, 61-73.

Steyvers, M., Shiffrin, R. M., \& Nelson, D. L. (2005). Word association spaces for predicting semantic similarity effects in episodic memory. In A. F. Healy (Ed.), Experimental cognitive psychology and its applications (pp. 237-249). Washington, DC: American Psychological Association.

Thomson, D. M., \& Tulving, E. (1970). Associative encoding and retrieval: Weak and strong cues. Journal of Experimental Psychology, 86, 255-262.

Tulving, E., \& Thomson, D. M. (1973). Encoding specificity and retrieval processes in episodic memory. Psychological Review, 80, 352-373.

\section{NOTE}

1. When WAS-REM was plotted as a function of forward strength with a high value for number of dimensions (469), it consistently overestimated probability of cued recall at each level of forward strength. This problem may have occurred because absolute strength was used as the index of associative strength: forward + backward + mediated strength. The fit might have been better with a relative index of associative strength that incorporated cue and target competitor strength: (forward + backward + mediated strength $) \div$ (forward + backward + mediated strength + cue competitor strength + target competitor strength). 


\section{APPENDIX \\ PIER2 and a Computational Example}

Studying a word initiates two processing activities, one automatic and implicit and one controlled and explicit. The target word $\mathrm{T}$ implicitly activates its representation $t$, which activates the representations of its associates in working memory. The activation of $t$ also initiates explicit encoding operations (e.g., rehearse, rate concreteness). The strengths of the implicit $S(\mathrm{I})$ and explicit $S(\mathrm{E})$ processing operations contribute independently to produce implicitly activated and episodically encoded representations of the target. The probability of recalling the target on an immediate test is

$$
\operatorname{Pr}\left(\mathrm{T} \mid \mathrm{I}_{t}, \mathrm{E}_{t}\right)=S\left(\mathrm{I}_{t}\right)+S\left(\mathrm{E}_{t}\right)-S\left(\mathrm{I}_{t}\right) S\left(\mathrm{E}_{t}\right)
$$

Because of the independence assumption, the model allows the two representations to have either additive or interactive effects. In contrast, an intersection version of PIER2, defined as $\operatorname{Pr}\left(\mathrm{T} \mid \mathrm{I}_{t}, \mathrm{E}_{t}\right)=S\left(\mathrm{I}_{t}\right) S\left(\mathrm{E}_{t}\right)$, would predict interactive effects between $S\left(\mathrm{I}_{t}\right)$ and $S\left(\mathrm{E}_{t}\right)$; for example, the explicit representation is recovered only if the implicit representation is recovered. Results showing that implicit and explicit representations can have additive effects would be inconsistent with the intersection version of the model.

Implicit cue-target strength in the extralist cuing task is computed as

$$
S\left(\mathrm{Q}_{q}, \mathrm{~T}_{\mathrm{I}}\right)=S_{q t} S\left(\mathrm{I}_{t}\right)+S_{q q} S\left(\mathrm{I}_{q}\right),
$$

where $q \neq t, S_{q t}$ is forward cue-to-target strength from free association norms, $S\left(\mathrm{I}_{t}\right)$ is implicit activation strength of the target, $S_{q q}$ is cue-to-cue self strength (nominally, 1.0), and $S\left(\mathrm{I}_{q}\right)$ is implicit activation strength of the cue. Shared associate and mediated strengths also contribute to implicit cue-target strength but are ignored in the following because they were not varied in these experiments. In Equation A2, $S\left(\mathrm{I}_{t}\right)$ and $S\left(\mathrm{I}_{q}\right)$ are defined as

$$
S\left(\mathrm{I}_{t}\right)=S_{t t}+\sum_{i}^{n} S_{t i}+\sum_{i}^{n} S_{i t}+\sum_{i}^{n} \sum_{j}^{n} S_{i j}
$$

where $t \neq i \neq j, S_{t t}$ is target self-strength (nominally 1.0 ), $S_{t i}$ is target-to-associate- $i$ strength, $S_{i t}$ is associate- $i$-totarget strength (resonance), and $S_{i j}$ is associate- $i$-to-associate- $j$ strength (connectivity).

Equation A3 assumes that the implicit activation strength of the target increases as an additive function of the strength of preexisting links in its network and that each link is reliably indexed by free association norms. For example, as is shown in Table A1, the target produces free associates $A_{1}-A_{6}$ at probabilities of $.25, .24, .20, .18$, .06 , and .04 , respectively. Associate 1 produces the target at .23, $A_{5}$ and $A_{6}$ at .15, and the remaining associates at 0 , and so forth. Given the values in the association matrix in Table A1, the result of computing Equation A3 is

$$
\begin{aligned}
S\left(\mathrm{I}_{t}\right) & =S_{t t}+\sum_{i}^{n} S_{t i}+\sum_{i}^{n} S_{i t}+\sum_{i}^{n} \sum_{j}^{n} S_{i j} \\
& =1.00+(.25+.24+.20+.18+.06+.04)+(.23+.05+.41+.22+.06)+(.15+.15+.15+.15) \\
& =3.54
\end{aligned}
$$

Note that targets with stronger links to, from, and among their associates will be more highly activated than those with fewer and weaker links. Because each link's strength adds to net activation strength, the model assumes that neither source, destination, nor direction affects net target activation strength. The total amount of activation is all that matters.

Table A1

Association Matrix

\begin{tabular}{lrrrrrrr}
\hline & Target & $A_{1}$ & $A_{2}$ & $A_{3}$ & $A_{4}$ & $A_{5}$ & $A_{6}$ \\
\hline Target & 1.00 & .25 & .24 & .20 & .18 & .06 & .04 \\
Associate 1 $\left(A_{1}\right)$ & .23 & & & & & .15 & .15 \\
Associate 2 $\left(A_{2}\right)$ & .05 & .15 & & & & & \\
Associate 3 $\left(A_{3}\right)$ & & & & & & & \\
Associate 4 $\left(A_{4}\right)$ & .41 & & .15 & & & & \\
Associate 5 $\left(A_{5}\right)$ & .22 & & & & & & \\
Associate 6 $\left(A_{6}\right)$ & .06 & & & & & & \\
\hline
\end{tabular}

The associates of the target are also activated to higher levels, depending on the strengths of the links providing the input. The strength of any associate is determined by adding the input from the target to the input from other associates in the set. The implicit activation strength of the target associate that becomes the test cue is

$$
S\left(\mathrm{I}_{q}\right)=S_{t q}+\sum_{j}^{n} S_{j q}
$$

where $q \neq j, S_{t q}$ is target-to-associate- $q$ strength, and $S_{j q}$ is associate-j-to-associate- $q$ strength. Note that $S\left(\mathrm{I}_{q}\right)=0$ when $S_{t q}=0$. When the test cue is not an associate of the target, backward target-to-cue strength is assigned a value of 0 . In Table A2, if Associate 1 becomes the test cue, the result is

$$
S\left(\mathrm{I}_{q}\right)=S_{t q}+\sum_{j}^{n} S_{j q}=.25+.15=.40 .
$$


APPENDIX (Continued)

Table A2

Retrieval Matrices

\begin{tabular}{|c|c|c|}
\hline Cue-Target Retrieval & $S\left(\mathrm{I}_{t}\right)$ & $S\left(\mathrm{I}_{q}\right)$ \\
\hline \multicolumn{3}{|c|}{ Strong Forward-Strong Backward Strength ( $A_{1}$ As Cue $)$} \\
\hline Target $(t)$ & $3.54^{\mathrm{a}}$ & $.40^{\mathrm{b}}$ \\
\hline Test cue $(q)$ & $.23^{\mathrm{c}}$ & $1.00^{\mathrm{d}}$ \\
\hline \multicolumn{3}{|c|}{$\begin{array}{l}S\left(\mathrm{Q}_{q}, \mathrm{~T}\right)=(.23 * 3.54)+[1.00 *(.25+.15)]=1.21 \\
\quad \operatorname{Pr}\left(\mathrm{T} \mid \mathrm{Q}_{q}\right)=1.21 \div\left[(1.21)+\left(.20^{\mathrm{e}}+.20^{\mathrm{f}}\right)\right]=.75\end{array}$} \\
\hline \multicolumn{3}{|c|}{ Strong Forward-Weak Backward Strength ( $A_{5}$ As Cue) } \\
\hline Target $(t)$ & $3.54^{\mathrm{a}}$ & $.21^{\mathrm{b}}$ \\
\hline Test cue $(q)$ & $.22^{\mathrm{c}}$ & $1.00^{\mathrm{d}}$ \\
\hline \multicolumn{3}{|c|}{$\begin{array}{c}S\left(\mathrm{Q}_{q}, \mathrm{~T}\right)=(.22 * 3.54)+[1.00 *(.06+.15)]=.99 \\
\operatorname{Pr}(\mathrm{T} \mid \mathrm{Q}, \mathrm{C})=.99 \div\left[(.99)+\left(.20^{\mathrm{e}}+.20^{\mathrm{f}}\right)\right]=.71\end{array}$} \\
\hline \multicolumn{3}{|c|}{ Weak Forward-Strong Backward Strength ( $A_{2}$ As Cue) } \\
\hline Target $(t)$ & $3.54 \mathrm{a}$ & $.39 \mathrm{~b}$ \\
\hline Test cue $(q)$ & $.05^{\mathrm{c}}$ & $1.00^{\mathrm{d}}$ \\
\hline \multicolumn{3}{|c|}{$\begin{array}{l}S\left(\mathrm{Q}_{q}, \mathrm{~T}\right)=(.05 * 3.54)+[1.00 *(.24+.15)]=.57 \\
\quad \operatorname{Pr}\left(\mathrm{T} \mid \mathrm{Q}_{q}\right)=.57 \div\left[(.57)+\left(.20^{\mathrm{e}}+.20^{\mathrm{f}}\right)\right]=.59\end{array}$} \\
\hline \multicolumn{3}{|c|}{ Weak Forward-Weak Backward Strength $\left(A_{6}\right.$ As Cue) } \\
\hline Target $(t)$ & $3.54^{\mathrm{a}}$ & $.19^{\mathrm{b}}$ \\
\hline Test cue $(q)$ & $.06^{\mathrm{c}}$ & $1.00^{\mathrm{d}}$ \\
\hline \multicolumn{3}{|c|}{$\begin{array}{l}S\left(\mathrm{Q}_{q}, \mathrm{~T}\right)=(.06 * 3.54)+[1.00 *(.04+.15)]=.40 \\
\quad \operatorname{Pr}\left(\mathrm{T} \mid \mathrm{Q}_{q}\right)=.40 \div\left[(.40)+\left(.20^{\mathrm{e}}+.20^{\mathrm{f}}\right)\right]=.50\end{array}$} \\
\hline
\end{tabular}

aEquation A3. bEquation A4. cForward strength indexed by free association norms. dTest cue strength. eTest cue competition strength. fTarget competition strength. Assumes that $A_{3}$ from the association matrix is not linked to the cue and became a target competitor.

Given these definitions, Equation A2 can be calculated. Table A2 illustrates the computations for strong and weak levels of forward and backward strengths. To calculate Equation A2, the predicted target activation level (Equation A3) is entered in the top row, first column of the retrieval matrix (3.54) and is multiplied by forward cue-to-target strength (.23), shown in the bottom row. The cue activation level (Equation A4) is entered in the top row, second column (.40) and multiplied by 1.0. The products of these multiplications are then summed to provide $S\left(\mathrm{Q}_{q}, \mathrm{~T}_{\mathrm{I}}\right)$.

Equation A2 calculates implicit cue-target strength and Equation A5 computes the probability of recalling the implicit representation of the target given the test cue relative to the noise produced by competing associates activated by the cue and by the target. Competing associates are those that fail to link the cue-target pairing within two associative steps, and they produce noise or error that reduces recall:

$$
\operatorname{Pr}\left(\mathrm{T}_{\mathrm{I}} \mid \mathrm{Q}_{q}\right)=\frac{S\left(\mathrm{Q}_{q}, \mathrm{~T}\right)}{S\left(\mathrm{Q}_{q}, \mathrm{~T}\right)+\sum_{q}^{n} A_{q}+\sum_{t}^{n} A_{t}}
$$

The calculation of Equation A5 is illustrated in Table A2 for the four combinations of high and low forward and backward strengths. In these calculations, we assume for convenience that a single cue competitor with strength .20 and a single target competitor with strength .20 contribute noise to the computations.

In the present experiments, recall was tested within approximately 2 min after study in order to provide the test instructions. Experiments 1 and 2 hold the explicit encoding operation constant, but Experiment 3 manipulates explicit encoding strength by varying level of processing and study time. Equation A6 adjusts recall for retention interval and type of intervening task and incorporates implicit and explicit encoding strength (Equation A1). It computes the probability of target recall in the presence of both extralist and context cues. This probability is computed as a power function of time engaged in distracting activities prior to testing:

$$
\operatorname{Pr}\left(\mathrm{T} \mid \mathrm{Q}_{q}, \mathrm{C}\right)=\operatorname{Pr}\left(\mathrm{T} \mid \mathrm{I}_{t}, \mathrm{E}_{t}\right) t^{-c},
$$

where $t$ is time in minutes $(t \geq 1)$ and $c$ is a context loss parameter that varies as a function of different types of disruptions. 